\title{
Reliability and Vulnerability Assessment of Interconnected ICT and Power Networks Using Complex Network Theory
}

DOI:

10.1109/PESGM.2018.8586596

\section{Document Version}

Accepted author manuscript

Link to publication record in Manchester Research Explorer

\section{Citation for published version (APA):}

Zhu, W., Panteli, M., \& Milanovic, J. V. (2018). Reliability and Vulnerability Assessment of Interconnected ICT and Power Networks Using Complex Network Theory. In 2018 IEEE Power and Energy General Meeting

https://doi.org/10.1109/PESGM.2018.8586596

\section{Published in:}

2018 IEEE Power and Energy General Meeting

\section{Citing this paper}

Please note that where the full-text provided on Manchester Research Explorer is the Author Accepted Manuscript or Proof version this may differ from the final Published version. If citing, it is advised that you check and use the publisher's definitive version.

\section{General rights}

Copyright and moral rights for the publications made accessible in the Research Explorer are retained by the authors and/or other copyright owners and it is a condition of accessing publications that users recognise and abide by the legal requirements associated with these rights.

\section{Takedown policy}

If you believe that this document breaches copyright please refer to the University of Manchester's Takedown Procedures [http://man.ac.uk/04Y6Bo] or contact uml.scholarlycommunications@manchester.ac.uk providing relevant details, so we can investigate your claim.

\section{OPEN ACCESS}




\title{
Reliability and Vulnerability Assessment of Interconnected ICT and Power Networks Using Complex Network Theory
}

\author{
Wentao Zhu, Student Member IEEE, Mathaios Panteli, Member IEEE, and J. V. Milanović, Fellow IEEE \\ School of Electrical and Electronic Engineering \\ The University of Manchester, Manchester, UK \\ wentao.zhu@manchester.ac.uk, mathaios.panteli@manchester.ac.uk, milanovic@manchester.ac.uk
}

\begin{abstract}
The increasing reliance of power systems on Information and Communication Technologies (ICTs) places higher requirements on the reliable and secure operation of ICT networks. This paper proposes an approach to measure the effect of failure/misoperation of ICT network components on the reliability of power grid. A new vulnerability index, namely the system security index (SSI), based on Complex Network Theory (CNT), is derived for this purpose. The vulnerability of each ICT network component is quantified based on the influence of its failure/misoperation on the probability of the failure/misoperation of the power system. The proposed approach is demonstrated on a real UK's power station intertrip scheme and compared with the conventional fault-tree analysis.
\end{abstract}

Index Terms-Information and Communication Technology (ICT), Complex Network Theory (CNT), reliability, vulnerability

\section{INTRODUCTION}

The power grids are going through significant transformation that requires new approaches to assess their vulnerability and assure secure and reliable operation. Interactions between physical (mostly power system based) and Information and Communication Technology (ICT) network elements of an ICT-electric power (ICT-EP) system are much more versatile and complex than in traditional systems. Therefore, new understanding and methods for assessing the reliability, as well as the interactions and interdependencies of this interconnected system as a whole must be developed, or the existing power system reliability assessment methods upgraded, to include the impact of ICT network components.

Model-based ICT-EP system reliability analysis is flexible and inexpensive to perform. A reliability block diagram (RBD) model to evaluate the reliability of electric power communication network was proposed in [1]. However, it does not quantify the vulnerability of the ICT component nor does it demonstrate its applicability on real networks. The direct ICT-EP interdependencies are analyzed in [2] using a state-mapping technique, which requires much computational power, and thus it is not feasible for larger network reliability analysis. A Monte Carlo and graph based simulation approach was proposed in [3] to study ICT's impact on power system, and its applicability was demonstrated on a PMU-based substation network. Fault trees $[4,5]$ and attack trees $[6,7]$ provide a straightforward visualization of ICT-EP logic relationships thus allowing potential events and causes of failures to be identified which helps to improve system reliability [8]. However, one of their main limitations is the exhaustive computational effort to enumerate all possible sequences of failures and algorithms to solve such models with computational time growing exponentially with the size of the system [9].

To overcome the limitations of conventional reliability analysis techniques and to enhance their applicability and scalability in complex system studies, this paper presents a novel and easy-to-implement approach to study the reliability of interconnected ICT and power systems. The interconnected system is first divided into two subsystems according to their functions, namely the power system and the ICT network. The reliability indices of each ICT network component are then computed using Markov modeling, and the results are incorporated into a complex network model to assess the overall interconnected system reliability, that is system security index $(S S I)$. Thereafter, the impact of each ICT network component's reliability on the normal operation of the interconnected system is assessed by calculating the increase in SSI when the studied ICT component is set to be completely reliable. Consequently, the vulnerabilities of ICT components are quantified. The approach is demonstrated on a real UK's power station intertrip scheme.

\section{RELIABILITY ANALYSIS AND VULNERABILITY ASSESSMENT}

Power system monitoring and control applications, such as Advanced Metering Infrastructure (AMI) and Demand Response (DR), require highly reliable ICT network. The failure of measurement units (e.g. phasor measurement units (PMUs)), communication channels, or the actuators, could therefore cause significant issues in power system operations. 


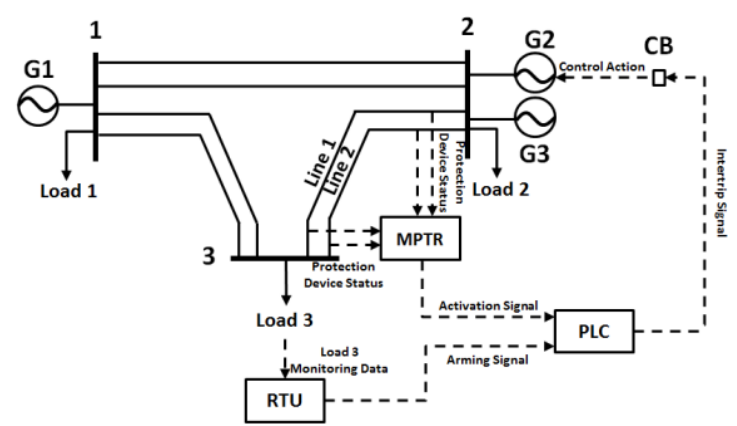

Fig. 1. Example diagram of protection system [10]

In an interconnected ICT-EP network, the ICT network nodes always function as intermediate ones to collect and transmit sensory data and to execute control command between electrical nodes which act as the information provider and command receiver at the head and the tail of the process, respectively. Fig. 1 presents an example of a generation rejection scheme (GRS) system structure [10], which will be used in this Section for demonstrating the proposed methodology. The purpose of this scheme is to maximize the power flow between bus 2 and bus 3 as the operating cost of the generator G1 is higher than the cost of the generators G2 and G3. Under the condition that the loading of bus 3 exceeds a certain level (monitored by the remote terminal unit (RTU)) and a fault exists on either line 1 or line 2 (detected by main protection trip relay (MPTR)), the scheme will be activated and a signal will be issued at programmable logic controller (PLC) to trip the circuit breaker of G2 and redispatch its output to G1. With GRS in place, the overloaded lines can be relieved, avoiding cascading failures of these lines.

\section{A. Interconnected ICT-EP network complex network model}

Complex network theory (CNT) allows the modelling of interconnected ICT-EP network as a graph $G$ which is composed of nodes $\left(V \equiv\left\{v_{1}, v_{2}, \ldots, v_{n}\right\}, n\right.$ is the number of nodes) and edges $\left(E \equiv\left\{e_{1}, e_{2}, \ldots, e_{\mathrm{m}}\right\}, m\right.$ is the number of edges). In this paper, nodes represent power systems' monitored or controlled circuits/generators and ICT network components. Nodes are then divided into two types $V_{e} \equiv\left\{v_{\mathrm{e} 1}\right.$, $\left.v_{\mathrm{e} 2}, \ldots, v_{\mathrm{ep}}\right\}$ and $V_{c} \equiv\left\{v_{\mathrm{cl} 1}, v_{\mathrm{c} 2}, \ldots, v_{\mathrm{cq}}\right\} \quad(p, q$ are the number of electrical and ICT network nodes respectively). To represent the source nodes which provide the power system measurement and the target nodes which receive the control action respectively, electrical nodes in $V_{e}$ are further divided into $V_{e s} \equiv\left\{v_{\text {est } 1}, v_{\text {es2 }}, \ldots, v_{\text {esa }}\right\}$ and $V_{e l} \equiv\left\{v_{\mathrm{et} 1}, v_{\mathrm{et} 2}, \ldots, v_{\mathrm{etb}}\right\}(a, b$ are the number of source and target nodes respectively). The interdependencies between power and ICT network components, being measurements, sensory and communication data, control command and control action are represented as directed edges connecting two or more dependent nodes. Fig. 2 shows a directed graph for the example protection system in Fig. 1.

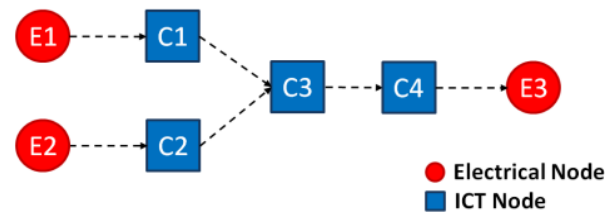

Fig. 2. Complex network model for example system
In this protection scheme, the monitored circuit power line 1 and line 2 are modelled as node E1, and load 3 is represented as node E2. Both E1 and E2 nodes work as source nodes to provide sensory data for the scheme to be activated. G2 is modelled as a target node E3 where the scheme finishes. The ICT network nodes $\mathrm{C} 1, \mathrm{C} 2, \mathrm{C} 3$, and $\mathrm{C} 4$ representing MPTR, RTU, PLC and CB respectively, between the source nodes E1 and E2 and the target node E3, provide important paths to allow the integrated protection action to take place. The collection of protection device status and load 3 monitoring data, activation signal, arming signal, intertrip signal, and control action are modelled as directed edges. Assuming these edges (i.e. communication links) are in perfectly reliable operating condition, any ICT node's misoperation will result in the failure of this protection scheme.

\section{B. ICT-EP Reliability Assessment Approach}

The fundamental design requirements for power system protection scheme are dependability, security, selectivity, and robustness [11]. Protection schemes may be made at the highest system level and the data may be provided from a wider area of the system [12], which causes a significant issue to ICT network components' security-based misoperations (SBMs). According to the NERC System Disturbance Reports from 1986-2009 [13], the majority of the disturbances related to protection misoperation mainly involved SBM (about 80\%) of protection schemes (including System Integrity Protection Schemes (SIPS)). In the example network of Fig. 1, the misoperation of any ICT network component may result in the spurious trip of generator 2, as shown in Fig. 3. Spurious trip here refers to the incorrect and unnecessary trip of the generator following a SBM of the protection scheme.

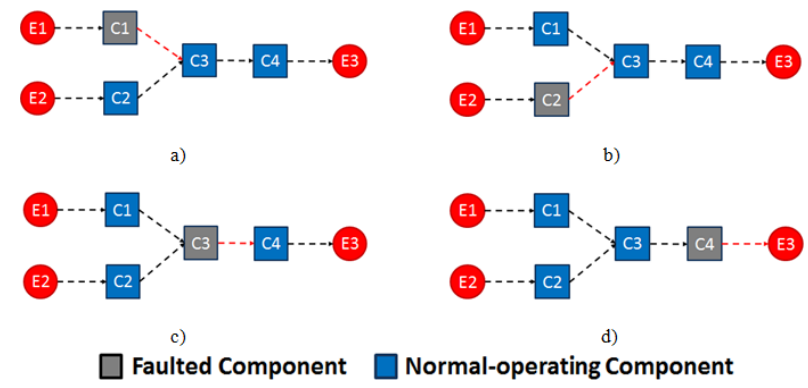

Fig. 3. SBM scenarios of example protection scheme

To quantify the security level of this system, or any interconnected ICT-EP network, i.e., the reliability of the system operating in a normal manner without any spurious trips of any power system components (e.g. lines and generators), a system security index (SSI) is defined as follows:

$$
S S I=\frac{1}{n_{e} n_{c}} \sum_{i \in V_{c} \cap V_{e S}, j \in V_{e t}, i \neq j} R_{i j}
$$

where $n_{e}$ and $n_{c}$ are the number of EP system nodes and ICT network nodes respectively. $R_{i j}$ is the reliability of the most reliable path between $i$ and $j$, which is calculated as

$$
R_{i j}=\prod_{c \in V_{c}}\left(1-P_{c}(S B M)\right)
$$


where $P_{c}(S B M)$ is the probability of security-based misoperation of ICT network component $c$ within the most reliable path between $i$ and $j$.

Assuming that the communication channels are $100 \%$ reliable, the No. 1 and 2 circuits protection devices status and load 3 monitoring data are correctly collected by PMR and RTU, and the $P_{c}(S B M)$ of ICT network components MPTR, RTU, PLC and CB are $0.1,0.2,0.2$ and 0.1 respectively, the $S S I$ of the example protection system is therefore estimated to be 0.34 , using equations (1) and (2). $n_{e}$ is equal to 3 and $n_{c}$ equals to 4 as shown in Fig. 2. The reliabilities $R_{i j}$ of the most reliable paths in the example protection scheme are presented in Table I. For example, the most reliable path between E1 and $\mathrm{E} 3$ is $\mathrm{E} 1 \rightarrow \mathrm{C} 1 \rightarrow \mathrm{C} 3 \rightarrow \mathrm{C} 4 \rightarrow \mathrm{E} 3$.

TABLE I ICT Paths Reliabilities of Example Network

\begin{tabular}{|c|c|c|}
\hline Beginning Node & Target Electrical Node & $\mathbf{R}_{\mathbf{i j}}$ \\
\hline E1 & E3 & 0.648 \\
\hline E2 & E3 & 0.576 \\
\hline C1 & E3 & 0.648 \\
\hline C2 & E3 & 0.576 \\
\hline C3 & E3 & 0.720 \\
\hline C4 & E3 & 0.900 \\
\hline
\end{tabular}

C. Probability of ICT network component security-based misoperation

The probability of security-based misoperation of the ICT network component $P_{C}(S B M)$ can be evaluated using Markov model as presented in Fig. 4, considering the detected failure and repair rates $(\lambda d d, \mu d d)$, the undetected failure and repair rates $\left(\lambda d u, \mu_{d u}\right)$ and the spurious trip and repair rates $\left(\lambda s, \mu_{s}\right)$ of each ICT component [14].

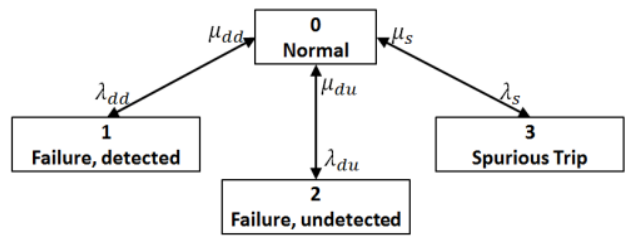

Fig. 4. Markov model of ICT system states [14]

The operating states include:

a) Normal state: All ICT network components operate in a secure manner.

b) Failure, detected: The dependability-based misoperation (DBM) of the ICT component is detected and fixed before it causes any problems, preventing overall DBM of the integrated system.

c) Failure, undetected: ICT components' DBM failure is not detected, resulting in the scheme failure to operate when needed.

d) Spurious trip: ICT network component(s) operate when not required, causing overall SBM.

Fig. 4 is used as a model to calculate $P_{C}(S B M)$ of ICT network components. As aforementioned, based on a review of the NERC disturbance reports [13], it was identified that the majority of misoperations (about $80 \%$ ) of protection schemes (including SIPS) are SBMs; hence the focus here is to evaluate the effect of SBM. However, the analysis can be extended to consider DBM too.

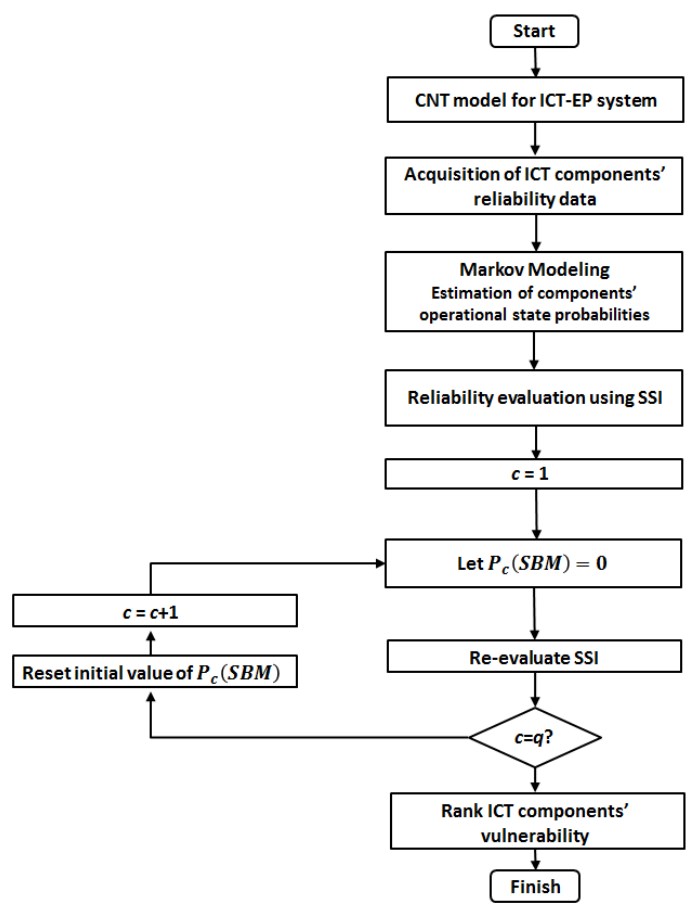

Fig. 5. Flow chart of the proposed method

\section{ICT network vulnerability analysis}

Fig. 5 presents the procedure for ranking the ICT network components' vulnerabilities related to SBM. First, the complex network model needs to be constructed. Using the reliability data of each ICT network component and the Markov model of Fig. 4, the $P_{C}(S B M)$ is estimated. Next, the reliability of the interconnected ICT-EP system is evaluated based on $S S I$. By setting the value $P_{C}(S B M)$ of each ICT network component $c$ to zero, the $S S I$ of the interconnected system is re-evaluated (denoted as $S S I^{*}$ ). The vulnerability of the ICT network component $V(C)$ is then assessed using (3) based on the difference between $S S I$ and $S S I^{*} . q$ is the total number of ICT components. Table II shows the $S S I^{*}$ and $V(C)$ of the example protection scheme of Fig. 2.

$$
V(C)=\left|\frac{S S I-S S I^{*}}{S S I}\right|
$$

TABLE II VulNerabilities of EXAmple Network ICT Components

\begin{tabular}{|c|c|c|}
\hline ICT Component & $S S I^{*}$ & $V(C)$ \\
\hline $\mathrm{C} 1$ & 0.351 & $3.2 \%$ \\
\hline $\mathrm{C} 2$ & 0.363 & $6.8 \%$ \\
\hline $\mathrm{C} 3$ & 0.405 & $19.1 \%$ \\
\hline $\mathrm{C} 4$ & 0.377 & $10.9 \%$ \\
\hline
\end{tabular}

\section{TEST SYSTEM -UK POWER STATION INTERTRIP SCHEME}

The proposed reliability and vulnerability assessment method is next demonstrated using an intertrip scheme, applied to a real hydro power station (represented as B3 in Fig. 6) in the UK, operated by the National Grid. 
The most critical circuits are the B7-B4 and B7-B5 circuits from an outage perspective. Only one supergrid connection is left between this supergrid and the rest of the system when an outage happens to either of these circuits, with a simultaneous outage of both B2-B4 circuits.

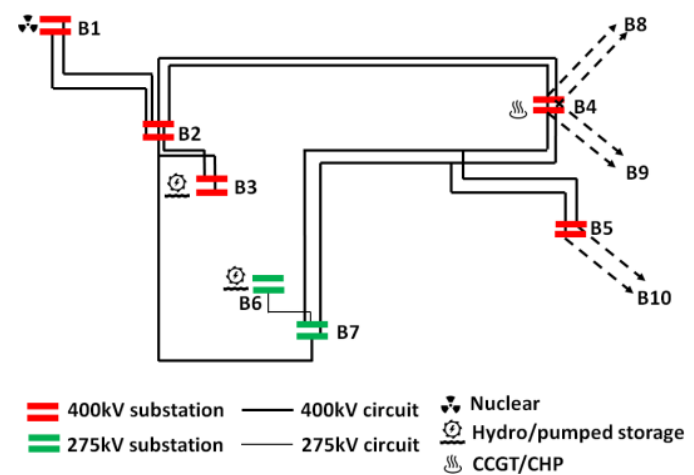

Fig. 6. Single-line diagram of supergrid in the UK

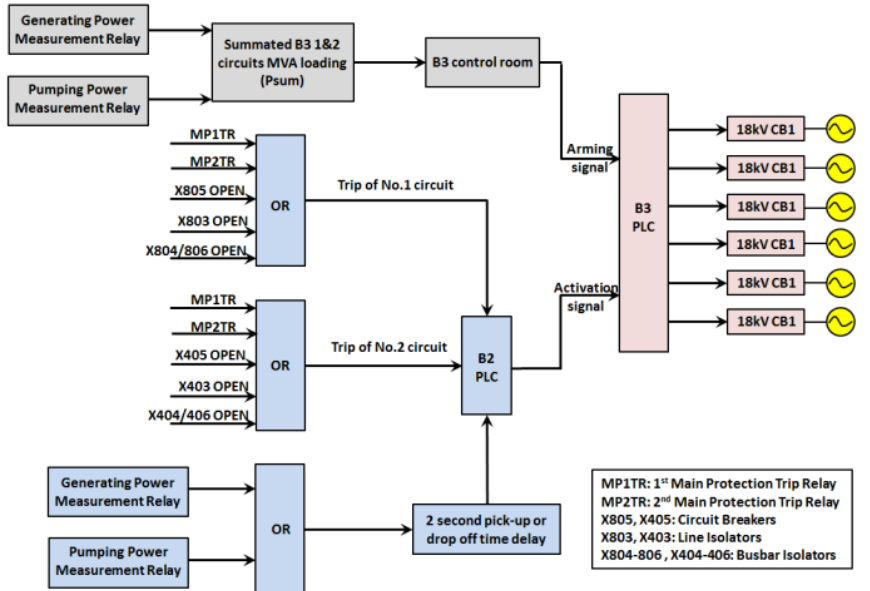

Fig. 7. Operating logic of intertrip scheme [14]

\section{A. Scheme operating logic}

There are three operational phases in the scheme: arming (grey), activation (blue) and implementation (red), as shown in Fig. 7. The scheme is manually switched in when the power transfer to or from B3 exceeds $1235 \mathrm{MW}$. The summated loading of the B3 1\&2 circuits, as shown in Fig. 7, are monitored by two PMRs located at B2, one each for pumping and generating. The generators located at B3 are only tripped when the generated or absorbed power is in excess of the levels which would lead to overloading of the transmission lines and/or instability of the supergrid shown in Fig. 6 [15].

The identification of the states of B2-B4 $1 \& 2$ circuits is based on the monitoring of the status of circuit breakers, line and busbar disconnectors associated with these circuits. The activation signal is sent from the $1^{\text {st }}$ and $2^{\text {nd }}$ main protection of B2-B4 $1 \& 2$ circuits (MP1TR and MP2TR) to cover stuck circuit breakers problems. Under the switch-in mode, the scheme is initiated when an intertrip signal is transmitted to B3, on the condition that both circuits are out concurrently. A short time delay was included in the scheme, to prevent spurious operation during system faults as a result of inaccurate measurements. Two PLCs at B2 and B3 are responsible for carrying out the scheme logic operations. The generators to be tripped are manually pre-determined by operating the selection switch located on the machine intertrip cubicle. Two generators are usually tripped by opening the appropriate $18 \mathrm{kV}$ circuit breakers connected to the motor of the generator on receipt of the activation signal. This ensures the remaining power transfer to/from B3 does not exceed 1235 MW, preserving the stability of the area.

\section{B. Complex network model of the intertrip scheme}

The interdependent complex network model of the intertrip scheme is presented in Fig. 8. On the electrical part, $\mathrm{B} 2-\mathrm{B} 4$ circuits and B3 generator groups are represented as electrical source node E1 and electrical target node E2 respectively. In the ICT network subsystem, the monitoring of B2-B4 No.1 and No.2 circuits, including the status of main protection trip relay (MPTR), $\mathrm{CBs}, \mathrm{BDs}$ and $\mathrm{LDs}$, are represented as ICT network nodes $\mathrm{C} 1$ and $\mathrm{C} 2$ separately. Nodes $\mathrm{C} 3$ and $\mathrm{C} 4$ represent respectively the generating and pumping PMRs located at B2. B2 PLC is represented as node $\mathrm{C} 5$, and $\mathrm{B} 3$ control room is represented as $\mathrm{C} 6$. The activation signal and arming signal are sent from B2 PLC and control room to B3 PLC (represented as node C7), to allow implementation signal to be issued. Nodes C8, C9 and C10 represent each probabilistically two random machines (15 combinations in total), since two generators are usually tripped when the scheme is activated and there are six generators located at B3.
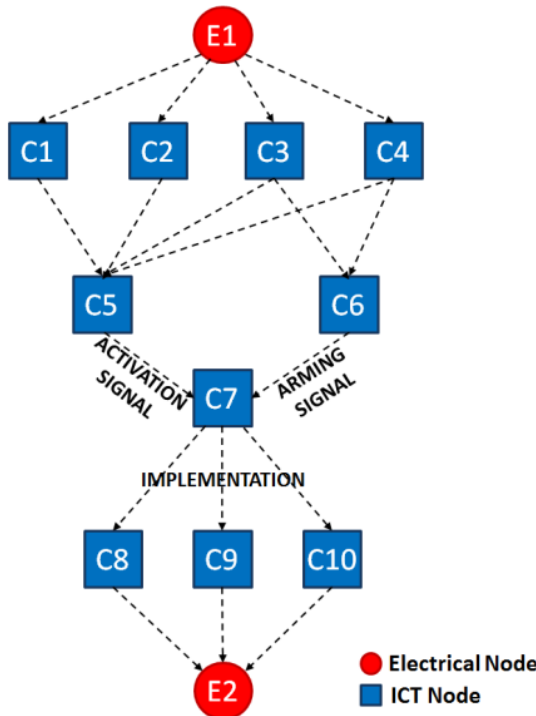

Fig. 8. Complex network model of intertrip scheme

\section{Intertrip scheme SSI}

The logic relationships of the scheme, as well as the reliability of each component are modelled as probabilistic weights in the graph. The proposed Markov model in Fig. 4 is applied to each ICT network component to estimate their operational state probabilities. Data of mean time to failure (MTTF) and mean time to repair (MTTR) are assumed based on experience and public databases and historical records $[16,17]$. The $P_{c}(S B M)$ of each ICT component is calculated using the MTTF and MTTR data and the Markov model, and they are presented in Table III [14]. Further details can be found in [14]. 
TABLE III $P_{C}(S B M)$ OF ICT NETWORK COMPONENTS

\begin{tabular}{|c|c|}
\hline Component & $\boldsymbol{P}_{\boldsymbol{c}}(\boldsymbol{S B} \boldsymbol{M})$ \\
\hline PMR & $5.2 \times 10^{-4}$ \\
\hline MPTR & $2 \times 10^{-4}$ \\
\hline CB & $1.8 \times 10^{-3}$ \\
\hline LD,BD & $1.22 \times 10^{-3}$ \\
\hline PLC & $6.7 \times 10^{-4}$ \\
\hline Human Error & $9.12 \times 10^{-5}$ \\
\hline
\end{tabular}

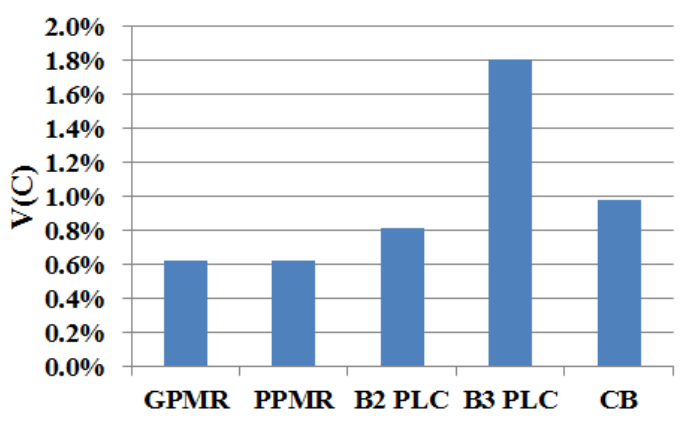

Fig. 9. ICT network components vulnerability index

Based on equations (1) and (2) and data presented in Table III, the SSI of the intertrip scheme is calculated to be 0.55 .

\section{Vulnerability analysis}

The vulnerability of each ICT network component related to the security-based misoperation of the system is calculated by the increase in system $S S I$ assuming each ICT component $c$ in perfectly reliable operating condition (i.e. $P_{c}(S B M)=0$ ). The results of vulnerabilities of the most critical ICT network components are presented in Fig. 9.

As can be seen from Fig. 9, the most vulnerable component is B3 PLC which collects the arming and activation signal, and implements the tripping command. The B2 PLC is also important as it needs to calculate and provide a reliable output from different information sources. However, it is less vulnerable than circuit breakers. The vulnerabilities of circuit breakers are mainly due to their positions in the logic relationships, as they are the closest component of initiating a spurious trip. PMRs rank the fourth in terms of SBM vulnerability, as they are merely information providers. The results are verified by the fault-tree analysis presented in [14].

\section{CONCLUSION}

Power system reliability analysis needs to consider the reliability of its supporting ICT network as modern power grid are becoming more and more dependent on the ICT network to fulfil its monitoring and control functions. The conventional reliability analysis techniques (e.g. Monte Carlo, fault-tree and attack-tree) require a significant amount of computational effort and therefore they are not practical for larger network analysis. Complex networks provide an alternative for ICT-EP system reliability analysis. The vulnerability of each component related to overall system reliability can be easily quantified, and the risk analysis and appropriate network improvement strategies can be proposed.

A system reliability index SSI is proposed in this paper to measure the security level of the system. Furthermore, the index facilitates the assessment of the vulnerability of each ICT network component. The results of the analysis using the newly proposed approach (i.e. based on complex network theory) confirm the findings of the past, conventional, faulttree analysis [18]; however the complex network model is found to be more scalable and easy-to-implement. The proposed methodology can be easily extended by including more logic relationships to facilitate analysis of large interconnected ICT-EP systems.

\section{REFERENCES}

[1] R. R. Zhang, Z. Y. Zhao, X. Chen, and J. Li, "Design and Implementation of a RBD-Based Algorithm for Reliability Analysis of Electric Power Communication Network," T\&D Asia: 2009 Transmission \& Distribution Conference \& Exposition: Asia and Pacific, pp. 710, 2009.

[2] B. Falahati, Y. Fu, and L. Wu, "Reliability Assessment of Smart Grid Considering Direct Cyber-Power Interdependencies," IEEE Transactions on Smart Grid, vol. 3, pp. 1515-1524, Sep 2012.

[3] Y. Zhang, M. Larsson, B. Pal, and N. F. Thornhill, "Simulation Approach to Reliability Analysis of WAMPAC System," 2015 IEEE Power \& Energy Society Innovative Smart Grid Technologies Conference (ISGT), 2015.

[4] H. Watson, Bell telephone laboratories. launch control safety study, Bell Telephone Laboratories, Murray Hill, NJ, USA, 1961.

[5] W. Vesely, F. Goldberg, N. Roberts, and D. Haasl, "Fault Tree Handbook," DTIC Document, Tech. Rep., 1981.

[6] B. Schneier, "Attack trees," Dr Dobbs Journal, vol. 24, pp. 21, Dec 1999.

[7] O. Sheyner, J. Haines, S. Jha, R. Lippmann, and J. M. Wing, "Automated generation and analysis of attack graphs," 2002 IEEE Symposium on Security and Privacy, Proceedings, pp. 273-284, 2002.

[8] V. Nagaraju, L. Fiondella, and T. Wandji, "A Survey of Fault and Attack Tree Modeling and Analysis for Cyber Risk Management," 2017 Ieee International Symposium on Technologies for Homeland Security (Hst), 2017.

[9] R. Gulati and J. B. Dugan, "A modular approach for analyzing static and dynamic fault trees," Annual Reliability and Maintainability Symposium - 1997 Proceedings, pp. 57-63, 1997.

[10] M. Panteli and P. Crossley, "Impact of SIPS performance on power systems integrity," 2011 International Conference on Advanced Power System Automation and Protection (APAP), 2011.

[11] M. Zima, "Special Protection Schemes in Electric Power Systems," EEH Power Systems Laboratory, Swiss Federal Institute of Technology in Zurich, Zürich, Switzerland 2002.

[12] "Network Protection \& Automation Guide: Protective Relays, Measurement \& Control," Alstom GridMay 2011.

[13] NERC, "System Disturbance Reports," 1986-2009.

[14] M. Panteli, P. A. Crossley, and J. Fitch, "Quantifying the reliability level of system integrity protection schemes," IET Generation Transmission \& Distribution, vol. 8, pp. 753-764, Apr 2014.

[15] K. Harker, "The North Wales Supergrid Special Protection Schemes," Electronics and Power, vol. 30, pp. 719-724, 1984.

[16] P. Hines, J. Apt, and S. Talukdar, "Large blackouts in North America: Historical trends and policy implications," Energy Policy, vol. 37, pp. 5249-5259, Dec 2009.

[17] T. R. Moss, The reliability data handbook. New York: ASME Press, 2005 . 